\title{
Consumer health information services cannot work alone
}

Consumer health information services offer valuable support to patients and relatives but an article in this issue (p 124) highlights a worrying gap between these services and health professionals. There is a danger that too much will be expected of those providing a health information service.

Entwistle and Watt describe a survey of consumer health information services carried out to explore opinions about, and use of, an information leaflet summarising the research evidence about the effectiveness of screening for prostate cancer. They found that the consumer health information services made good use of the leaflet but staff were making decisions about who should or should not receive the leaflet and patients were going back to them for further advice and information.

As the authors point out, these services do a good job on limited resources, but the training they have received does not equip them to provide expert advice. Indeed, a report of a training programme to help consumer health information services staff make sense of evidence about effectiveness commented that the training workshops "cannot turn critical appraisal novices into experts". ${ }^{1}$

Not only that but the information service staff are generalists with a broad remit and are unlikely to be able to provide in depth advice to back up the material they give out in response to the many different types of enquiries they receive. Consumer self help groups are better equipped to provide people with specialist information about a particular condition or treatment and information service staff should be working in cooperation with them.

The most important source of expert advice, however, should be the health professional. Unfortunately there is still great variation among clinicians in their awareness of sources of good quality information for patients. The government funded Centre for Health Information Quality, which was set up in $1997^{2}$ will no doubt eventually begin to have some impact, but at the moment very few clinicians seem to be using consumer health information services in a way which benefits patients.

If the health professional does not volunteer information at the time of consultation and the patient subsequently has to seek it out, the patient is unlikely to return to the professional for further advice on the issue because they may well think that the information they have obtained does not have his or her approval.

Yet general practitioners and other health professionals could improve matters fairly easily by being more willing to acknowledge that patients and relatives need information. This does not have to take up a great deal of their time. They could provide patients with the telephone number of a consumer health information service or use it as a resource. For example, they could use it to obtain up to date material related to common conditions and to enquire about material on rarer conditions as and when they occur. The patient could be given the opportunity of asking further questions or discussing the issues when they have had time to read the material. With the health professional acting as the link between the consumer health information services and patients in most cases, they could ensure that the patient received expert advice when they needed it.

If general practitioners and other health professionals took a more active role in providing information to patients and relatives, there would also be more chance that everyone who needed information would benefit instead of only those knowledgeable enough to use the consumer health information services. Research has shown that non-English speakers in particular, have difficulty getting the information they need to understand their illness, the decisions that have to be made, and how they can manage their condition. ${ }^{3}$

In the end, it is in the health professional's best interests to provide good information to patients. There is evidence to show that patients who are well informed comply more readily with treatment. ${ }^{4}$ Perhaps more importantly, if patients do not receive the information they need from well coordinated health providers and health information services, they will increasingly turn to the Internet where the quality of information is uncontrolled. Without the support of health professionals in interpreting this information, patients may decide on the best course of action for themselves and in so doing, may come into conflict with those providing services. ${ }^{5}$

SHIRLEY MCIVER

Senior fellow, Health Services Management Centre,

University of Birmingham

1 Milne R, Oliver S. Evidence-based consumer health information: developing teaching in critical appraisal skills. Int $\mathcal{F}$ Qual Health Care 1996;8:439-45

2 Centre for Health Information Quality ( $\mathrm{CH}$ HiO). Highcroft, Romsey Road, Winchester, Hampshire SO22 5DH, UK. Telephone 01962863511 extenWinchester.

3 Anderson J. Empowering patients: issues and strategies, Soc Sci Med 1996; 43:697-705.

4 Kaplan SH,Greenfield S, Ware JE, Assessing the effects of physician-patient interaction on the outcomes of chronic disease. Medical Care 1989;27:11027.

5 Coiera E, The Internet's challenge to healthcare provision. BMf 1996;312: 